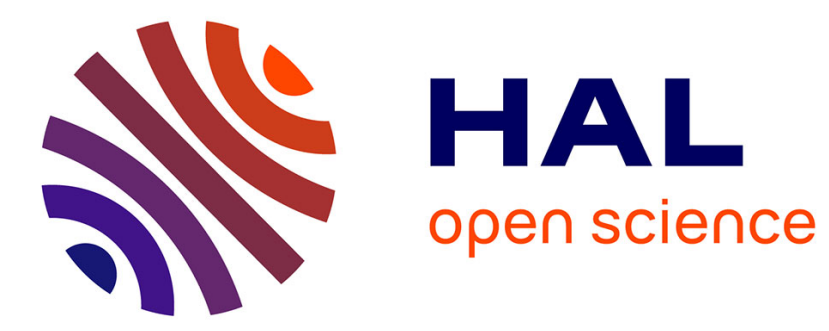

\title{
The optical behaviour of slightly ferroelectric BaTiO3
}

F.J. Bartis

\section{To cite this version:}

F.J. Bartis. The optical behaviour of slightly ferroelectric BaTiO3. Journal de Physique Lettres, 1985, 46 (7), pp.335-339. 10.1051/jphyslet:01985004607033500 . jpa-00232520

\section{HAL Id: jpa-00232520 https://hal.science/jpa-00232520}

Submitted on 1 Jan 1985

HAL is a multi-disciplinary open access archive for the deposit and dissemination of scientific research documents, whether they are published or not. The documents may come from teaching and research institutions in France or abroad, or from public or private research centers.
L'archive ouverte pluridisciplinaire HAL, est destinée au dépôt et à la diffusion de documents scientifiques de niveau recherche, publiés ou non, émanant des établissements d'enseignement et de recherche français ou étrangers, des laboratoires publics ou privés. 
Classification

Physics Abstracts

78.20

\title{
The optical behaviour of slightly ferroelectric $\mathrm{BaTiO}_{3}$
}

\author{
F. J. Bartis \\ Department of Physics, Indiana University, Bloomington, Indiana 47405, U.S.A.
}

(Reçu le 24 mai 1984, révisé les 20 novembre 1984 et 28 janvier 1985, accepté le 11 février 1985)

\begin{abstract}
Résumé. - Un champ fluctuant est produit par les oscillations des dislocations relatives aux petits domaines ferroélectriques sur leurs côtés. Par l'effet Kerr quadratique ce champ cause une déviation en $T /\left(T-T_{0}\right)$ de l'indice de réfraction par rapport à sa variation linéaire à haute température et des anomalies en $T /\left(T-T_{0}\right)^{2}$ des coefficients photoélastiques et électrooptiques.

Abstract. - A fluctuating field is produced by the oscillations of dislocations relative to the small ferroelectric domains at their sides. Through the quadratic Kerr effect this field causes a $T /\left(T-T_{0}\right)$ deviation of the refractive index from its linear variation at high temperature and $T /\left(T-T_{0}\right)^{2}$ anomalies of the electrooptic and photoelastic coefficients.
\end{abstract}

\section{Introduction.}

Some thought must now be given to alternatives to the classic soft-mode description of ferroelectric phase transformations. Detailed studies of $\mathrm{SbSI}$ and $\mathrm{BaTiO}_{3}$ indicate that the harmonic modes of the lattice provide only a small fraction of the static electric permittivity just above the Curie point [1,2]. Grigas and his associates propose a soft anharmonic mode of the lattice to account for the microwave dielectric resonance observed in SbSI above its ferroelectric transition [3]. Here we invoke a soft defect mode to explain some of the optical properties of $\mathrm{BaTiO}_{3}$ above $120^{\circ} \mathrm{C}$.

Our starting point is a simple physical picture of this defect mode. We envisage a straight edge dislocation with a cylindrical domain of the ferroelectric phase at its side. In the mode of interest the whole dislocation line oscillates with respect to the ferroelectric domain. The ferroelectric phase is retained on the dilated side of the dislocation because the stresses there cause the crystallites to pass from the ferroelectric to the paraelectric state well above the nominal transition point at atmospheric pressure. To gauge the persistence of the ferroelectric phase we take the crystal lattice to be an isotropic elastic continuum. Thus the stresses around a dislocation are found to go inversely as the distance. Assuming that the transition temperature varies linearly with the stress, we obtain a $\left(T-T_{0}\right)^{-1}$ dependence for the radius of the ferroelectric domain. Here $T_{0}$ denotes the transition point at zero stress. For our formal description of the defect mode we consider a small displacement of the dislocation line relative to the ferroelectric domain. As a rough estimate of the change in the elastic potential energy per unit length of the dislocation we obtain

$$
W \approx G b f s^{2} / 2 \pi R_{n}
$$


where $G$ is the shear modulus, $b$ is the length of the Burgers vector of the dislocation, $f$ is the fractional change of volume in passing from the paraelectric to the ferroelectric state and the displacement $s$ is supposed to be small compared to the domain radius $R_{n}$. Differentiating with respect to the displacement, we see that the dislocation is subject to a linear restoring force [4]. So, if it is displaced and then released, it will undergo simple harmonic motion with an angular frequency $\omega_{\mathrm{e}}$ given by

$$
\omega_{\mathrm{e}}^{2} \approx G f / \pi^{2} \rho b R_{n},
$$

where $\rho$ is the density of $\mathrm{BaTiO}_{3}$ [5]. Putting in the temperature dependence of the domain radius, we discover that the frequency goes as $\left(T-T_{0}\right)^{1 / 2}$. In line with current practice we refer to this low-frequency oscillation as the soft mode of dislocations.

\section{Theory.}

Above $120^{\circ} \mathrm{C}$ thermal excitation of such a low-energy mode is very likely. It is clear from the equipartition theorem that, as the angular frequency decreases, the oscillation amplitude $\phi$ increases :

$$
\omega_{\mathrm{e}}^{2} \phi^{2} \propto T .
$$

Here $T$ is the ambient temperature on the absolute scale. Because dislocations in ionic $\mathrm{BaTiO}_{3}$ have charged cores encircled by oppositely charged sheaths, their thermal agitation is sensed at large distances through a fluctuating electric field. In a first approximation the fluctuating field $F$ is proportional to their amplitude of oscillation. It follows that

$$
F^{2} \propto T / \omega_{\mathrm{e}}^{2} .
$$

Inserting the temperature variation of the angular frequency, we find that near $T_{0}$ the longrange fluctuating electric field grows in strength :

$$
F^{2} \propto T /\left(T-T_{0}\right) .
$$

Through the quadratic Kerr effect the fluctuating field modifies the way light propagates through a $\mathrm{BaTiO}_{3}$ crystal. Assuming that the dislocation structure of the crystal has cubic symmetry, we predict that the principal refractive indices deviate from their normal values by the same amount :

$$
n_{0}-n=B T /\left(T-T_{0}\right)
$$

where $B$ denotes a constant.

We can readily imagine what happens at the microscopic level when a static electric field is applied to the crystal. The domains with electric polarizations parallel to the external electric field grow, while those with polarizations antiparallel contract. In turn, the dislocations allied to these domains alter their frequencies and amplitudes of oscillation. Now the deviation of the refractive index from its normal value is different for light polarized in the direction of the static field.

What electrooptic and photoelastic coefficients tell us is how effective a static electric field $E$ and external strain $x$ are in changing the inverse optical permittivity :

$$
\delta\left(1 / n^{2}\right)=r E+p x .
$$

Subjecting the crystal to just an incremental electric field, we obtain the electrooptic coefficient in the form

$$
r=\left(-2 / n^{3}\right)(\delta n / \delta E)
$$


To evaluate the ratio of increments we use relation (6) under the assumption that the external field does not alter the normal refractive index. Recalling that the nominal transition point varies linearly with the strength of a static electric field, we determine that the electrooptic coefficient is anomalous near $T_{0}$ :

$$
n^{3} r \propto T /\left(T-T_{0}\right)^{2} .
$$

Applying just an incremental strain to the crystal, we get the photoelastic coefficient in the form

$$
p=\left(-2 / n^{3}\right)(\delta n / \delta x) .
$$

As an external strain produces a proportional shift in the nominal transition point, the photoelastic coefficient should also be anomalous near $T_{0}$ :

$$
n^{3} p \propto T /\left(T-T_{0}\right)^{2} .
$$

In passing we note that the refractive index of $\mathrm{BaTiO}_{3}$ shows a variation of less than $0.1 \%$ between 120 and $240^{\circ} \mathrm{C}$. So we commit a very small error, when we drop the index from relation (9) or (11).

\section{Discussion.}

The present theory compares favorably with earlier attempts to explain the optical behaviour of $\mathrm{BaTiO}_{3}$. Its obvious strong point is a greater predictive power. Wemple and DiDomenico contend that an external strain modifies the contribution of critical polarization fluctuations to the refractive index [6]. Through the fluctuation-dissipation theorem they are able to relate the photoelastic coefficients to a correlation volume and a derivative of the static electric permittivity with respect to the strain. Taking the correlation volume to be independent of the temperature, they arrive at the observed $\left(T-T_{0}\right)^{-2}$ dependence of the photoelastic coefficient. Unfortunately they do not indicate why the correlation volume is constant here, whereas it increases dramatically near an ordinary critical point.

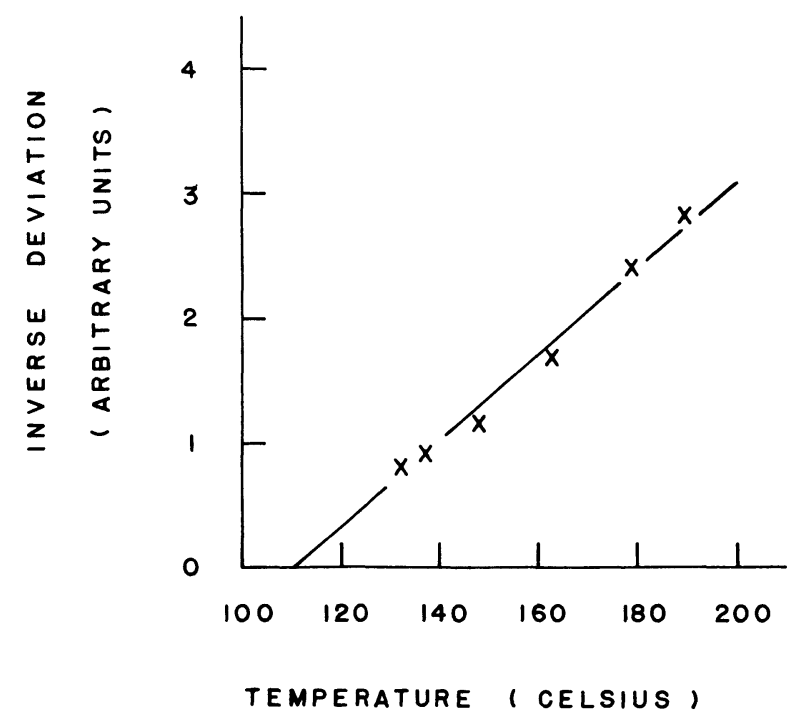

Fig. 1. - Determination of $T_{0}$ from the deviation of the refractive index $n$ from its normal value $n_{0}$. The inverse deviation or $\left(n_{0}-n\right)^{-1}$ is plotted against the temperature. 
Burns and Dacol also invoke critical fluctuations of the electric polarization to account for the deviation of the refractive index from its high-temperature behaviour [7]. They claim that polarization fluctuations are important some hundred degrees above the nominal transition point. However, when the Ginzburg criterion is applied, the ferroelectric transition is found to have a critical region of only two-tenths of a degree [8].

The deviation of the refractive index from its normal value has a specific temperature dependence if the transition is mediated by the soft mode of dislocations. According to relation (6) the deviation should roughly follow a Curie-Weiss law. To test this prediction we use the data published by Burns and Dacol [7]. The normal value of the refractive index is obtained through a linear extrapolation of the data at high temperature. In the accompanying figure the inverse deviation is plotted against the temperature. The scatter of the points along a straight line indicates that for $\lambda=4579 \AA$ the deviation goes approximately as $\left(T-T_{0}\right)^{-1} . T_{0}$ is of course the intersection of the line with the abscissa, i.e. $110^{\circ} \mathrm{C}$.

It is satisfying to find that analyses of the electrooptic and photoelastic behaviour of $\mathrm{BaTiO}_{3}$ yield the same $T_{0}$, within the experimental uncertainties $[9,10]$. From the measurements of, say, the electrooptic coefficient $r$ a plot of $\left(n^{3} r\right)^{-1 / 2}$ versus the temperature is constructed. The coefficient diverges as $\left(T-T_{0}\right)^{-2}$ if the points fall along a straight line. Surprisingly, the electrooptic measurements of reference [9] seem to give a more precise value of $T_{0}$ than the refractive measurements of reference [7]. Although the electrooptic data are for $\lambda=6328 \AA, T_{0}$ is still $110^{\circ} \mathrm{C}$.

In closing we want to comment on the scope of the present theory. Here we are concerned with just a few of the optical properties of $\mathrm{BaTiO}_{3}$. The underlying physical picture can however be profitably applied to several others. For purposes of illustration, let us consider the elastic scattering of light. Presumably, a $\mathrm{BaTiO}_{3}$ crystal is made optically inhomogeneous by the presence of small ferroelectric domains in a paraelectric matrix [11]. Since the ferroelectric domains are cylindrical, the Rayleigh intensity is roughly proportional to their size. Physically we expect the domain size to be determined by the stress field of the oscillating dislocations. Because the relaxation time of the interphase boundary is very large compared to the oscillation period of the dislocations, the domain size can be evaluated as if the dislocations remain at their equilibrium positions. Levanyuk and his colleagues have already shown how static defects influence the elastic scattering of light during a second-order phase transition [12, 13]. Our theory enables us to consider the way both static and dynamic defects affect the scattering of light during the first-order ferroelectric transition of $\mathrm{BaTiO}_{3}$. The fluctuating field $F$ of the dislocations varies over distances comparable to the wavelength. So, if $F$ contributes to the refractive indices in the manner contemplated here, then the birefringence should vary a little from one point to another in the crystal. Light should therefore experience small rotations of its plane of polarization to the right as well as to the left. Accepting this picture, we are not surprised by the report that light acquires a depolarized component on passing through a $\mathrm{BaTiO}_{3}$ crystal above $120^{\circ} \mathrm{C}[14]$.

\section{Acknowledgments.}

We thank John Bomburas and the Physics Department of the Massachusetts Institute of Technology for their kind hospitality during the revision of the manuscript.

\section{References}

[1] Grigas, I. and Belyatskas, R., Fiz. Tverd. Tela 20 (1978) 3675.

[2] Luspin, Y., Servoin, J. L. and Gervais, F., J. Phys. C 13 (1980) 3761.

[3] Kalesinskas, V., Grigas, I., Paprotnyi, V. and Spitsyna, V., Fiz. Tverd. Tela 25 (1983) 738. 
[4] Bartis, F. J., J. Physique Lett. 40 (1979) L-351.

[5] Bartis, F. J., J. Physique Lett. 41 (1980) L-161.

[6] Wemple, S. H. and DiDomenico, M. Jr., Phys. Rev. B 1 (1970) 193.

[7] BURns, G. and DaCol, F. H., Ferroelectrics 37 (1981) 661.

[8] Benguigui, L., J. Phys. C 8(1975) 17.

[9] Sinyakov, E. V., Golovyanko, A. A. and Savchenko, V. G., Kristallografiya 16 (1971) 553.

[10] Cohen, M. G., DiDomenico, M. Jr. and Wemple, S. H., Phys. Rev. B 1 (1970) 4334.

[11] Romanovskis, T. and Zvirgzde, J., Phys. Stat. Sol. A 32 (1975) K41.

[12] Ginzburg, V. L., Levanyuk, A. P. and Sobyanin, A. A., Phys. Rep. 57 (1980) 151.

[13] Levanyuk, A. P., Osipov, V. V., Sigov, A. S. and Sobyanin, A. A., Zh. Eksp. Teor. Fiz. 76 (1979) 345.

[14] Zvirgzde, J. A., Zvirgzde, J. V. and Fritsberg, V. J., J. Phys. Soc. Jpn, Suppl. B 49 (1980) 166. 